\title{
REINFORCEMENT OF ELASTIC POLYMER MATRIX FILLED WITH ULTRAFINE PARTICLES
}

\author{
M. KRYSZEWSKI \\ Center of Molecular and Macromolecular Studies, Polish Academy of Sciences \\ Sienkiewicza 112, 90-363 Łódź, Poland \\ AND G.W. BĄK \\ Institute of Physics, Technical University of Eódź \\ Wólczańska 219, 93-005 Eódź, Poland
}

(Received July 1, 1997; in final form September 22, 1997)

\begin{abstract}
The nanofiller of noble metal may modify the properties of the polymernoble metal composite even if the concentration of the metal nanoparticles is very low. The aim of this paper is to study the influence of colloidal particles in nanocomposites. For our studies we have selected polymethyl methacrylate-palladium nanocomposite. It is shown that the effective radius of the immobilised matrix environment is much greater than the radius of the palladium particles. Due to the binding of the matrix polymer and the palladium surface some volume of the surrounding matrix becomes immobilised giving rise to the effective increase in the size of the palladium filler. The analysis of a few models of nanocomposites used for interpretation of the mechanical properties has also been presented.
\end{abstract}

PACS numbers: 62.20.Dc, 81.20.Ti

\section{Introduction}

The importance of inhomogeneous and multiphase systems has increased significantly during the last decades. First of all it refers to many composite materials that are widely used in many fields of technology. In the recent years a special attention has been paid to polymer composites containing fine noble metal nanoparticles because they may exhibit a variety of unusual properties. The nanofiller of noble metal may modify the properties of the polymer-noble metal composite even if the concentration of the metal nanoparticles is very low.

There are many methods of preparation of solid sols of various metals, particularly noble metals. We will only mention two of them which seem to be typical examples of new trends in that field independently of classical colloid route. The first one [1] consists in decomposition of organic salts of noble metals (or ion 
pairs of metal salts) during polymerization of acrylic polymers and postheating. Metal-polymer nanocomposites can be obtained from a variety of organometallic precursors by dissolving them in supercritical fluid (SCF), e.g., $\mathrm{CO}_{2}$ and infused into solid polymer. Chemical or thermochemical reduction to the base metal occurs either by presence of SCF solution or subsequent removal of the solution by depressurization which also produces nanoscale domains within polymer matrix. The high permeation rate of $\mathrm{CO}_{2}$ in virtually all polymers as well as the wide range of metallic reagents, which are soluble in $\mathrm{CO}_{2}$, render this technique generally useful.

In most of two-component composite materials the interphase originating at the boundary between two basic components often plays a significant role and have remarkable influence on the macroscopic properties of a composite. In the case of ageing in humid environment a thin electrolyte layer between the filler and the matrix usually arise. This comparatively well conducting electrolyte layer gives rise to a decrease in mechanical properties and its physical properties may be investigated using broad band dielectric spectroscopy $[2,3]$. In fact the electrolyte layer is the additional third quite different phase in a composite.

Another kind of interphase exists in these composite materials in which the matrix environment of the filler particles is partially immobilised due to the interaction between the surface of the filler and the matrix. In this situation every filler particle is surrounded by the modified matrix material giving rise to the effective increase in size of the filler particle.

The aim of this work is to study the influence of colloidal particles in nanocomposites on their modulus of elasticity. For our studies we have selected polymethyl methacrylate-palladium nanocomposite which may be obtained according to the method described by Nakao [4].

\section{Experimental}

The transparent polymethyl methacrylate (PMMA) nanocomposites were obtained according to the method described in Nakao's work [4]. Acetylacetanato palladium(II), at different concentrations, was dissolved in methyl methacrylate containing benzoyl peroxide as an initiator of polymerization. The samples were polymerised by heating at $70^{\circ} \mathrm{C}$ for about $60 \mathrm{~min}$. The resulting viscous solution was transferred between two glass plates separated by a teflon spacer and heated again at $45^{\circ} \mathrm{C}$ for $24 \mathrm{~h}$. The plates of PMMA prepared in such a way could be postheated for $1 \mathrm{~h}$ at a higher temperature (about $120^{\circ} \mathrm{C}$ ) to study the coloration for optical investigations which will be not discussed here. TEM of the colored PMMA samples indicated well-dispersed ultrafine particles of palladium of the diameter between 1 and $2 \mathrm{~nm}$. The elastic modulus was determined at the room temperature by a dynamic mechanical analyser.

\section{Results and discussion}

Many papers concerning the physical properties of polymer composites suggest that the interphase existing at the boundary between the filler and the matrix has a significant influence on the properties of these systems. There are two groups of models describing the interphase. Most of the models assume that the interphase substance is uniform [4-8]. However another model assuming the non-uniform 
physical properties of the interphase has also been put forward [9-11]. The former one has been used for interpretation of the thermal expansion of some polymer composites. The model assumes that some physical quantities of the interphase are given by $P Q_{i}=a r^{2}+b r+c$, where $P Q_{i}$ denotes either the thermal expansion coefficient, the elastic modulus or the Poisson ratio of the interphase substance. $r$ is the distance from the center of the filler particle, $a, b$ and $c$ are constants. The size of the interphase is defined by $\mathrm{d} P Q_{i} / \mathrm{d} r=0$. The model has been successfully used for explanation of results concerning the thermal expansion of some polymer composites [10] including metal-filled epoxies [9]. According to the model both the interphase volume fraction and the interphase thickness initially increase with the increasing volume fraction of the filler showing a maximum, after which both the quantities decrease down to comparatively low values.

However the applicability of the non-uniform model of the interphase seems limited to the comparatively large filler particles. For instance, the diameter of the filler particles reported for glass-filled epoxy resin was over $10 \mu \mathrm{m}$ [12]. In our case the diameter of the metal particles is about $1 \mathrm{~nm}$ and the diameter of the interphase region is estimated to be of some nanometers. In this situation this is justified to assume that the physical properties of the interphase region are either uniform or nearly uniform and the models assuming uniform physical properties of the interphase region will be used for interpretation of the results. Figure 1 shows a model of the network structure arising around a palladium particle. Due to the binding of the matrix polymer and the palladium surface some volume of the surrounding matrix becomes immobilised giving rise to the effective increase in the size of the palladium filler.

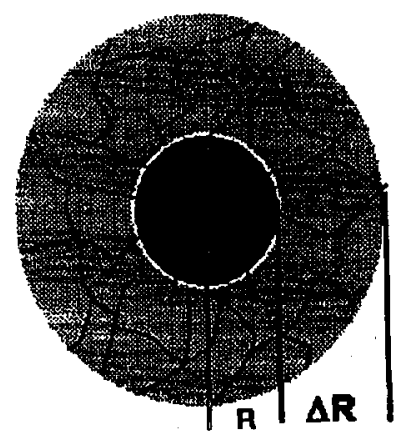

Fig. 1. Model of network structure around palladium nanoparticles in methacry-late/palladium composite. $R$ - the radius of palladium particle, $\Delta R$ - the effective increase in the particle size due to the interaction of the palladium surface with the matrix.

As it is mentioned above there are a few models for description of the mechanical properties of composite materials [4-8] but none of them turns out to be applicable for a wide range of composites. Most of the models provide only some experimental formula for the modulus of elasticity of composite $E_{c}^{\prime}$, but the equations of Kerner seem to be derived from the very basic considerations of me- 
chanical properties of a complex composite system. Despite this, the agreement between the equations and the experimental data is usually not satisfactory. It has been suggested that the reason for that may be the fact that the effective volume fraction of partially immobilised material $\Phi_{e}$ is greater than the volume fraction of the filler $\Phi_{\mathrm{f}}$ [13]. If we assume that $\Phi_{\mathrm{e}}$ is given by [13]

$$
\Phi_{\mathrm{e}}=\Phi_{\mathrm{f}}\left(1+\frac{\Delta R}{R}\right)^{3},
$$

then the agreement between the theoretical description and the experimental results may be achieved.

Three various equations for the modulus of elasticity have been used to describe the data obtained for polymethyl methacrylate-palladium composite. The first one results directly from Kerner's equation for Poisson ratio $\nu=0.5$ if the elastic modulus of the filler $E_{\mathrm{f}}^{\prime}$ is much greater than that of the matrix $E_{0}^{\prime}$

$$
\frac{E_{\mathrm{c}}^{\prime}}{E_{0}^{\prime}}=1+\frac{2.5 \Phi_{\mathrm{f}}}{1-\Phi_{\mathrm{f}}}
$$

The second one results from the series-parallel model of two-phase composite material

$$
\frac{E_{\mathrm{c}}^{\prime}}{E_{0}^{\prime}}=1+\frac{\Phi_{\mathrm{f}}}{1-\Phi_{\mathrm{f}}^{1 / 3}}
$$

and the third one is a result of the so-called parallel model

$$
\frac{E_{\mathrm{c}}^{\prime}}{E_{0}^{\prime}}=1+\frac{E_{\mathrm{f}}^{\prime} \Phi_{\mathrm{f}}}{E_{0}^{\prime}} \text {. }
$$

The experimental results for the polymethyl methacrylate-palladium composite are shown in Table.

\section{TABLE}

Elastic modulus of polymethyl methacrylate/palladium composite.

\begin{tabular}{c|c}
\hline $\begin{array}{c}\text { Volume fraction } \\
(\%)\end{array}$ & $\begin{array}{c}\text { Elastic modulus } \\
\text { [GPa] }\end{array}$ \\
\hline 0 & 1.9 \\
0.004 & 3.4 \\
0.007 & 4.4 \\
0.012 & 5.6
\end{tabular}

The first two equations (i.e., Eqs. (3) and (4)) rather cannot be used for the interpretation of the obtained results because the increase in the elastic modulus is too small (see Fig. 2). The experimental values are in good agreement with the theoretical curve resulting from Eq. (4) provided that $\Delta R=22.5 \AA$ is used. The theoretical curve was fitted by changing the increase in the effective radius $\Delta R$ as it is shown in Fig. 3 and the agreement was obtained just for the value $22.5 \AA$. This may suggest that the effective radius of the immobilised matrix environment is much greater than the radius of the palladium particles which was about $10 \AA$. 


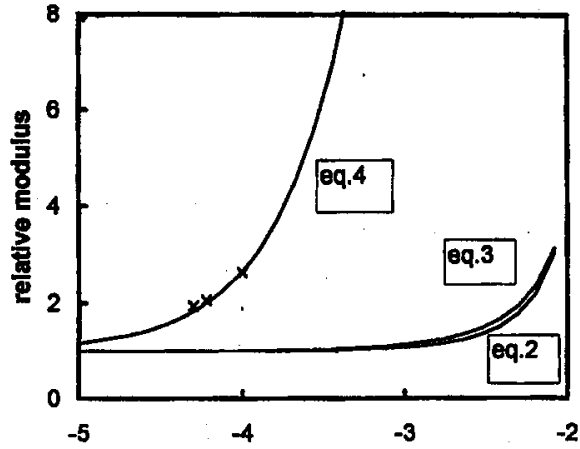

Fig. $2 \log$ (volume fraction)

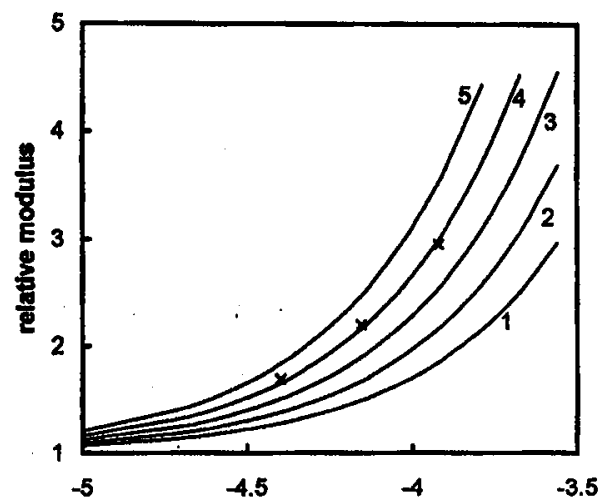

Fig. $3 \quad \log$ (volume fraction)

Fig. 2. Relative elastic modulus as a function of volume fraction predicted by Eqs. (2), (3) and (4). $x$ - experimental data. $\Delta R=22.5 \AA$.

Fig. 3. Relative elastic modulus as a function of volume fraction for various increase in the palladium filler particle size. (1) $\Delta R=15 \AA$, (2) $\Delta R=17.5 \AA$, (3) $\Delta R=20 \AA$, (4) $\Delta R=22.5 \AA,(5) \Delta R=25 \AA . \times$ - experimental points.

\section{Conclusions}

The following conclusions may be drawn from the above results:

1. Volume of the immobilised environment of palladium nanoparticles in polymethyl methacrylate matrix gives rise to the increase in the effective volume fraction of the filler.

2. Measurements of the elastic modulus may enable us to estimate the size of the matrix environment bound with the filler surface.

\section{References}

[1] J. Ulański, M. Kryszewski, in: Encyclopedia of Advanced Materials, Eds. D. Bloor, R.J. Brook, M.C. Flemings, S. Mahojan, R.W. Cahn, Pergamon, Oxford 1994, p. 2301.

[2] J.J. Watkins, T.J. McCarthy, ACS PMSE 73, 158 (1995).

[3] M. Kryszewski, G.W. Bakk, Polish J. Chem. 70, 1 (1996).

[4] Y. Nakao, J. Chem. Soc. Chem. Commun. 10, 826 (1993).

[5] H. Eilers, Kolloid-Z. 97, 313 (1941).

[6] Y. Sato, O. Furukawa, Rubber Chem. Technol. 36, 1081 (1963).

[7] L. Nielsen, J. Appl. Polym. Sci. 10, 97 (1966).

[8] U. Zorll, J. Appl. Polym. Sci. 10, 1315 (1966).

[9] E. Kerner, Proc. R. Soc. Lond. B 69, 808 (1956).

[10] G.C. Papanicolau, P.S. Theocaris, Colloid Polym. Sci. 257, 239 (1979).

[11] G.C. Papanicolau, D. Bakos, K. Imielinska, private communication.

[12] E.P. Sideridis, G.C. Papanicolau, Rheol. Acta 27, 608 (1988).

[13] K. Ziegel, J. Appl. Polym. Sci. 17, 1119 (1973). 